\title{
Safety and efficacy of early radiofrequency catheter ablation in patients with paroxysmal atrial fibrillation complicated with amiodarone-induced thyrotoxicosis
}

\author{
Maojing Wang ${ }^{1}$, Shanglang Cai ${ }^{1}$, Libo Sun ${ }^{1}$, Qing Zhao ${ }^{1}$, Wenjing Feng ${ }^{2}$ \\ ${ }^{1}$ Department of Cardiology, The Affiliated Hospital of Qingdao University, Qingdao, China \\ ${ }^{2}$ Department of Geriatric Medicine, The Affiliated Hospital of Qingdao University, Qingdao, China
}

\begin{abstract}
Background: Amiodarone is an antiarrhythmic drug that is frequently used to control atrial fibrillation $(A F)$. Many patients with $A F$ are afraid of the risk of ablation and take amiodarone, some patients develop amiodarone-induced thyrotoxicosis (AIT). The purpose of the study was to investigate the safety and efficacy of early radiofrequency catheter ablation in patients with paroxysmal AF complicated with AIT.

Methods: From the 146 consecutive patients with paroxysmal AF who had been treated with amiodarone and underwent 3-dimensional mapping system guided circumferential pulmonary vein isolation (PVI) at our center from January 2013 to June 2014, 20 had developed AIT. Thirty controls with normal thyroid function and matched for baseline characteristics were selected.
\end{abstract}

Results: Pulmonary vein isolation was completed in all patients without serious complications and with similar procedural (170.60 \pm 14.80 vs. $158.18 \pm 9.06 \mathrm{~min} ; p=0.062)$ and $X$-ray exposure $(16.48 \pm 2.15$ vs. $15.36 \pm 1.57 \mathrm{~min} ; p=0.058)$ time in AIT vs. control groups; however, upon coronary sinus catheter pacing (from $300 \mathrm{~ms}$ to $200 \mathrm{~ms}$ ) after intravenous isoproterenol administration 30 min post PVI, rates of induction of AF (35\% vs. $3.33 \%$; $p=0.005)$ and of non-pulmonary vein-related atrial tachyarrhythmias (50\% vs. 6.67\%; $p=0.01)$ were higher, while those for atrial flutter (15\% vs. 3.33\%; $p=0.17)$ and atrial tachycardia (15\% vs. $6.67 \% ; p=0.31$ ) were similar, as was the recovery of conduction of pulmonary vein potential (15\% vs. 30\%; $p=0.191$ ). In AIT vs. control group, atrial tachyarrhythmia recurrence rate was higher at 3 months $(45 \%$ vs. $16.67 \%, p=0.032)$ but not between 3 and 12 months (30\% vs. 23.33\%; $p=0.418$ ) follow-up.

Conclusions: Early catheter ablation for paroxysmal AF in patients with AIT appeared safe and effective albeit with higher atrial tachyarrhythmia recurrence rate up to 3 months but not beyond 12 months after PVI relative to controls. (Cardiol J 2016; 23, 4: 416-421)

Key words: amiodarone-induced thyrotoxicosis, atrial fibrillation, catheter ablation, safety, efficacy

Address for correspondence: Wang Maojing, MD, Department of Cardiology, The Affiliated Hospital of Qingdao University, 16 Jiangsu Road, Qingdao City, Shandong Province 266003, China, fax: 86-0532-82911598, e-mail: wangmaojing@sina.com 


\section{Introduction}

Amiodarone is frequently used to control atrial fibrillation (AF) - the most common arrhythmia $[1,2]$. Because each amiodarone molecule contains two iodine atoms with about $10 \%$ dissociating into free iodine [3], long-term use of amiodarone may lead to abnormal thyroid function in $15-20 \%$ of patients [4], seriously impairing further drug treatment for AF. Although paroxysmal AF refractory to oral antiarrhythmic drugs is a class I indication of catheter ablation now commonly used for maintenance of sinus rhythm [5], studies are lacking on its safety and efficacy in patients with frequent and symptomatically intolerable paroxysmal AF complicated with amiodarone-induced thyrotoxicosis (AIT), which is the subject of the present study.

\section{Methods}

\section{Case selection}

From 146 consecutive patients with paroxysmal AF as per the 2014 American Heart Association/American College of Cardiology/Heart Rhythm Society (AHA/ACC/HRS) guideline who underwent ablation at the Affiliated Hospital of Qingdao University from January 2013 to June 2014, 20 (11 males/9 females; mean age $63.65 \pm$ \pm 8.91 years) with frequent and symptomatically intolerable paroxysmal AF complicated with AIT were selected as the AIT group, while other 30 (17 males/13 females; mean age $60.67 \pm 8.54$ years) who also received oral amiodarone but continued to have normal thyroid function were randomly selected as the control group. AIT was diagnosed in patients who had normal thyroid function and after taking amiodarone had a thyroid stimulating hormone (TSH) level $<0.27$, free thyroxine (FT4) level $>22$, and/or free triiodothyronine (FT3) level > 6.8 (normal values, TSH: $0.27 \sim 4.2 \mathrm{IU} / \mathrm{mL}$; FT4: $12.0 \sim 22.0 \mathrm{pmol} / \mathrm{L}$; FT3: $3.10 \sim 6.80 \mathrm{pmol} / \mathrm{L}$ ) [6]. Patients with any of the following conditions were excluded from the AIT group: AF found at the same time or after thyrotoxicosis occurrence; evidence of organic thyroid disease in thyroid ultrasound; presence of antithyroid peroxidase (ATPO) antibody and anti-thyroglobulin antibody (TG-Ab); persistent AF; structural heart diseases, such as valvular heart disease and congenital heart disease; and presence of left atrial thrombus in transesophageal echocardiography. Before taking amiodarone, all patients underwent laboratory testing for FT3, FT4, TSH, ATPO, TG-Ab, and thyroid ultrasound; while on amiodarone, thyroid function was checked every 3 months; amiodarone was withdrawn as soon as thyrotoxicosis was found, and catheter ablation was planned; all patients (including AIT and control groups) stopped amiodarone and received pulmonary vein isolation (PVI) in 3 days; 4 patients with severe thyrotoxicosis received methimazole before ablation and the other 16 patients in AIT group did not receive any treatment. Before circumferential pulmonary vein isolation (CPVI) all patients: underwent laboratory testing for FT3, FT4, TSH, ATPO, and TG-Ab $48 \mathrm{~h}$ pre procedure; received the anticoagulant warfarin, which was not stopped before PVI if the international normalized ratio (INR) was between 2 and 3 ; and were assessed $24 \mathrm{~h}$ pre procedure with esophageal ultrasonography to exclude left atrial thrombus.

All the patients had signed a general written informed consent. Ethical approval was given by the medical Ethics Committee of the Affiliated Hospital of Qingdao University. The study protocol conformed to the ethical guidelines of the 1975 Declaration of Helsinki.

\section{Mapping and ablation}

After left subclavian vein puncture, a 10 -electrode electrophysiology catheter was placed in the coronary sinus (CS); after 2 atrial septal punctures, two 8.5 F Swartz long sheaths (SL1) were placed in the left atrium; changes in pulmonary vein potential were recorded with a LASSO catheter; under the guidance of the Carto system, a $3.5 \mathrm{~mm}$ ablation catheter (Navi-Star ThermoCool, Biosense-Webster, USA) was used for left atrial reconstruction and CPVI of the bilateral pulmonary veins; at the anterior wall, the discharge energy was $35 \mathrm{~W}$; at the top and rear walls, the discharge energy was $30 \mathrm{~W}$; at each ablation point, the discharge lasted $20 \mathrm{~s}$ or the potential was decreased by $80 \%$; and the 2-way bilateral PVI was performed as previously published [7, 8]. After CPVI, the patient was observed for $30 \mathrm{~min}$; isoproterenol via intravenous drip then was given to increase the heart rate up to $120 \mathrm{bpm}$. Burst artial pacing was performed from CS at a circle length from $300 \mathrm{~ms}$ to $200 \mathrm{~ms}$ to observe whether there was atrial tachycardia, atrial flutter, or AF episodes, and whether there occurred recovery of conduction ( $\mathrm{RoC}$ ) of pulmonary vein potential.

\section{Postoperative care and follow-up}

After catheter ablation, oral warfarin was prescribed for at least 3 months, with dose adjustment to maintain the INR between 2 and 3 . 
Anticoagulation was extended beyond 3 months if AF relapsed. After PVI, none of the patients received any antiarrhythmic drugs. For patients in the AIT group with severe thyrotoxicosis, upon consultation with endocrinologists, methimazole was given. The following parameters were captured: (1) Perioperative complications such as cardiac tamponade, embolization, and hematoma; (2) Operation time and X-ray exposure time; (3) Whether arrhythmia was induced and whether there was RoC of pulmonary vein potential after isoproterenol administration; (4) The recurrence rate of atrial tachyarrhythmia after PVI: surface electrocardiogram (ECG) and dynamic ECG were recorded at each month during 12-month followup or at the time the patient perceived recurrence to determine if it was symptomatic AF, typical atrial flutter, or other atrial tachyarrhythmia. $\mathrm{AF}$ was defined according to 2014 AHA/ACC/HRS guidelines. We have defined typical atrial flutter as cavotricuspid isthmus-dependent atrial flutter, atrial rate $250-350 / \mathrm{min}$. Other atrial tachyarrhythmia was defined as atypical atrial flutter or atrial tachycardia. The former refers to non-cavotricuspid isthmus-dependent atrial flutter, atrial rate 250$-350 / \mathrm{min}$; the latter refers to atrial tachyarrhythmia with atrial rate $150-250 / \mathrm{min}$, whose mechanism is reentrant or focal. Recurrence could be defined if a monitored episode of atrial arrhythmia lasted over $30 \mathrm{~s}$. If the recurrence occurred within 3 months after PVI, it was defined as early recurrence and if beyond 3 months as late recurrence [9].

\section{Statistical analysis}

All the continuous variables are expressed as mean \pm standard deviation and were compared with single factor analysis of variance, while categorical variables are expressed as number and percentage and were compared using the $\chi^{2}$ test. Statistical analyses were performed using SPSS19.0 statistical software. $\mathrm{P}<0.05$ was considered statistically significant.

\section{Results}

Distribution of baseline sociodemographic and clinical characteristics, including gender, age, left atrial dimension, AF duration, and procedural characteristics (Table 1).

In the AIT group, isoproterenol infusion $30 \mathrm{~min}$ after PVI induced: 1 ) AF in 7 (35\%) cases: 3 from the superior vena cava (SVC) terminated after SVC isolation [10], 2 were associated with RoC of pulmonary vein conduction, and were terminated by ablation at areas with RoC along the PVI ablation line, and 2 were neither associated with the SVC nor with $\mathrm{RoC}$ of pulmonary vein conduction, and were treated with electrical cardioversion; 2) Atypical atrial flutter or atrial tachycardia in $3(15 \%)$ cases: 1 left atrial roof-dependent macroreentrant flutter, which was terminated by linear ablation at the left atrial roof, validated by 2 -way block of atrial top line [11, 12]; 1 mitral isthmus-dependent macroreentrant flutter, which was terminated by linear ablation at the mitral isthmus, with the ablation being continued until there was 2 -way block of the mitral isthmus line [11, 12]; and 1 associated with atrial-pulmonary vein potential conduction gap, which was terminated by gap ablation along the PVI ablation line; 3) Typical atrial flutter in $3(15 \%)$ cases, all tricuspid isthmus-dependent which were treated with tricuspid isthmus ablation. In the AIT group, 3 (15\%) cases showed recovery of atrium-pulmonary vein potential conduction: 2 cases led to AF and 1 case led to atrial tachycardia.

In the control group, AF from SVC was induced in $1(3.33 \%)$ case, which was terminated after SVC isolation; atrial tachycardia from the gap along the PVI ablation line was induced in $2(6.67 \%)$ cases, which were terminated after gap ablation; typical atrial flutter was induced in 1 case, which was treated with tricuspid isthmus ablation. There were $9(30 \%)$ cases of atrium-pulmonary vein potential conduction recovery, in which atrial tachycardia was induced in 2 cases, and no atrial arrhythmia was induced in 7 cases.

Non-pulmonary vein-related atrial tachyarrhythmia was significantly more often induced in the AIT group (10 [50\%] cases: 5 AF, 3 atrial flutter, and 2 atrial tachycardia cases) than in the control group (1 AF [3.33\%] case). Early but not late recurrence rate of atrial tachyarrhythmia was significantly higher in the AIT than in the control group, with significant differences at every month up to 3 months (Table 2).

Before PVI, the levels of thyroid hormones were as follows: FT3 (minimum 6.47, maximum 13.57 , average $7.78 \pm 1.61 \mathrm{pmol} / \mathrm{L})$, FT4 (minimum 22.53, maximum 60.26 , average $30.43 \pm$ $\pm 8.23 \mathrm{pmol} / \mathrm{L}$ ), and TSH (minimum 0.005 , maximum 0.261 , average $0.116 \pm 0.091 \mathrm{IU} / \mathrm{mL}$ ). Among the 20 patients with AIT, 4 patients with severe AIT were treated with methimazole as per endocrinologist's advice after PVI. Thyroid function was higher at the time of ablation, and returned to normal in 1,2 , and 1 cases at 1,2 , and 3 months after PVI, respectively. In other 16 patients who did not take the anti-hyperthyroidism drug, thyroid 
Table 1. Comparison of clinical and procedural characteristics between the two studied groups.

\begin{tabular}{|c|c|c|c|}
\hline & $\begin{array}{l}\text { AIT group } \\
(n=20)\end{array}$ & $\begin{array}{l}\text { Control group } \\
(\mathrm{n}=30)\end{array}$ & $\mathbf{P}$ \\
\hline Male gender & $11(55 \%)$ & $17(56.67 \%)$ & 0.565 \\
\hline Age [years] & $63.65 \pm 8.91$ & $60.67 \pm 8.54$ & 0.24 \\
\hline Left atrial diameter $[\mathrm{cm}]$ & $4.29 \pm 0.43$ & $4.47 \pm 0.32$ & 0.91 \\
\hline Left ventricular end-diastolic dimension [cm] & $4.71 \pm 0.25$ & $4.47 \pm 0.32$ & 0.696 \\
\hline Left ventricular ejection fraction [\%] & $0.62 \pm 0.05$ & $0.64 \pm 0.04$ & 0.714 \\
\hline Duration of atrial fibrillation [months] & $12.75 \pm 12.31$ & $20.07 \pm 34.29$ & 0.413 \\
\hline Dose of received amiodarone [mg/d.] & 200 & 200 & 1 \\
\hline Time of received amiodarone [months] & $12.6 \pm 7.22$ & $11.35 \pm 8.62$ & 0.579 \\
\hline Cases of thyrotoxicosis treated & $4(20 \%)$ & $0(0 \%)$ & - \\
\hline Operation time [min] & $170.60 \pm 14.80$ & $158.18 \pm 9.06$ & 0.062 \\
\hline X-ray exposure time [min] & $16.48 \pm 2.15$ & $15.36 \pm 1.57$ & 0.058 \\
\hline Success rate of instant pulmonary vein isolation [\%] & $100 \%$ & $100 \%$ & - \\
\hline Atrial fibrillation induced intraoperatively & $7(35 \%)$ & $1(3.33 \%)$ & 0.005 \\
\hline $\begin{array}{l}\text { atypical atrial flutter or atrial tachycardia } \\
\text { induced intraoperatively }\end{array}$ & $3(15 \%)$ & $2(6.67 \%)$ & 0.31 \\
\hline Typical atrial flutter induced intraoperatively & $3(15 \%)$ & $1(3.33 \%)$ & 0.17 \\
\hline $\begin{array}{l}\text { Non-pulmonary vein-related atrial } \\
\text { tachyarrhythmias intraoperatively }\end{array}$ & $10(50 \%)$ & $2(6.67 \%)$ & 0.01 \\
\hline $\begin{array}{l}\text { Recovery of conduction of pulmonary vein } \\
\text { potential induced intraoperatively }\end{array}$ & $3(15 \%)$ & $9(30 \%)$ & 0.191 \\
\hline Cardiac tamponade & $0(0 \%)$ & $0(0 \%)$ & - \\
\hline Symptomatic cerebral embolism & $0(0 \%)$ & $0(0 \%)$ & - \\
\hline Hematoma & $1(5 \%)$ & $2(6.67 \%)$ & 0.651 \\
\hline
\end{tabular}

Data are presented as mean \pm standard deviation or $\mathrm{n}(\%)$; AIT — amiodarone-induced thyrotoxicosis

Table 2. Comparison of early (up to 3 months) and late (beyond 3 and up to 12 months) recurrence rates after pulmonary vein isolation between the amiodarone-induced thyrotoxicosis (AIT) group and the control group.

\begin{tabular}{lccc}
\hline Month & $\begin{array}{c}\text { AlT group } \\
(\mathbf{n}=20)\end{array}$ & $\begin{array}{c}\text { Control group } \\
(\mathbf{n}=30)\end{array}$ & $\mathbf{P}$ \\
\hline \multicolumn{4}{l}{ Early recurrence } \\
1 & $8(40 \%)$ & $4(13.33 \%)$ & 0.035 \\
2 & $9(45 \%)$ & $5(16.67 \%)$ & 0.032 \\
3 & $9(45 \%)$ & $5(16.67 \%)$ & 0.032 \\
Late recurrence & & \\
4 & $4(20 \%)$ & $3(10 \%)$ & 0.277 \\
5 & $4(20 \%)$ & $3(10 \%)$ & 0.277 \\
6 & $5(25 \%)$ & $4(13.33 \%)$ & 0.247 \\
7 & $5(25 \%)$ & $5(16.67 \%)$ & 0.355 \\
8 & $5(25 \%)$ & $5(16.67 \%)$ & 0.355 \\
9 & $6(30 \%)$ & $5(16.67 \%)$ & 0.221 \\
10 & $6(30 \%)$ & $5(16.67 \%)$ & 0.221 \\
11 & $6(30 \%)$ & $6(20 \%)$ & 0.315 \\
12 & $6(30 \%)$ & $7(23.33)$ & 0.418 \\
\hline
\end{tabular}

function returned to normal in 10, 4, and 2 cases at 1,2 , and 3 months after PVI, respectively, which is consistent with the report by Bartalena et al. [13] who documented that thyroid function returns to normal from 1 to 3 months in AIT patients [13-15].

\section{Discussion}

In the present study, 20 patients with paroxysmal AF complicated with AIT underwent radiofrequency ablation therapy to treat frequent symptomatic episodes, and as compared to matched controls with normal thyroid function, had significantly more often isoproterenol-induced $\mathrm{AF}$ and non-pulmonary vein-related atrial tachyarrhythmias, and significantly higher rates of early recurrence of atrial tachyaarhythmia.

Higher rates of isoproterenol-induced arrhythmias may be secondary to high thyroid hormone levels, which can: 1) Inhibit the inward L type calcium channel, increase the outgoing potassium current, shorten the action potential plateau period, and 
increase the repolarization speed, thereby shortening action potential duration, effective responding period, and reentrant wavelength in the atria, and resulting in tiny reentrant waves [16]; 2) Alter the atrial distribution of autonomic nerves, cause over-allocation of atrial sympathetic nerves and autonomic dysfunction, and increase atrial arrhythmia incidence; 3) Significantly increase the autorhythmicity of atrial evoked potential [17], causing AF as well as atrial flutter and atrial tachycardia.

The abovementioned features also might underlie the higher early recurrence rates of atrial tachyarrhytmia in the AIT group, which also might be related to local edema after CPVI because of the impact of ablation energy on local tissues. Bertaglia et al. [18] reported that within 3 months after ablation for patients with AF, $46 \%$ of patients had atrial arrhythmias, most of which spontaneously resolved 3 months later.

In order to decrease the recurrence rate of AIT patients, we spent more operation time in the AIT group (AIT group vs. control group, $170.60 \pm$ $\pm 14.80 \mathrm{~min}$ vs. $158.18 \pm 9.06 \mathrm{~min}$ ), which may result in lower $\mathrm{RoC}$ of the pulmonary veins when intravenously treated with isoprenaline. Nevertheless, no significant difference was found between the two groups $(p=0.191)$. In fact, all patients had no $\mathrm{RoC}$ of the veins at the moment the operation finished. We examined the rate of $\mathrm{RoC}$ of the pulmonary veins during operation, but the recurrence rate of atrial tachyarrhythmias in AIT patients was examined after operation. During the first 3 months, we cannot examine the rate of RoC after operation, the thyroid hormone levels can affect the percentage of recurrence.

Within 3 months, thyroid hormone levels were high and normalized thereafter. Thus, the high recurrence 3 months post ablation was likely due to residual mild hyperthyreosis. The thyroid hormone levels of both the AIT group and the control group were normal after 3 months post ablation, which resulted in similar recurrence.

The present study found that after 3 months post CPVI, when the thyroid function returned to normal, the late success rate was similar in both groups. Auer et al. [19] had reported an AF incidence of $13.8 \%$ in patients with primary hyperthyroidism, and 5 times that of the general population in patients with subclinical hyperthyroidism. When thyroid function returns to normal, the effects of the thyroid hormone on the atria fade away with most associated AF resolving [19]. In a previous study, for patients with primary hyperthyroidism, after thyroid function returned to normal but still had
$\mathrm{AF}$, ablation success rate was similar to that of $\mathrm{AF}$ patients without hyperthyroidism [20]. The latter result as well as those in the present study indicate that after thyroid hormone levels return to normal, $\mathrm{AF}$ is associated with only focal evoking loci.

In this study, after PVI, the late recurrence rate of atrial tachyarrhythmias was lower than early recurrence rate, and similar to that of paroxysmal AF patients with normal thyroid function. Possible reasons for the latter observation include: (1) In the AIT group, hyperthyroidism was not very severe, with thyroid function returning to normal in both 16 of 20 patients 3 months after amiodarone discontinuation or with addition of methimazole for 3 months in the remaining 4 with more severe AIT; (2) Intraoperative isoproterenol via intravenous drip induced atrial flutter, AF, and atrial tachycardia unrelated to pulmonary vein potential, reducing the relapse of atrial arrhythmia after catheter ablation [21]; (3) After PVI, observation for $30 \mathrm{~min}$, and isoproterenol via intravenous drip, the gap along the PVI ablation line was treated with ablation, reducing the atrial tachyarrhythmias secondary to recovery of atrium-pulmonary vein conduction [21].

\section{Limitations of the study}

Interpretation of the findings in the present study is limited by its single-center, retrospective design with a small sample size and by the fact that hyperthyroidism was mild among studied patients with paroxysmal AF complicated with AIT. Recurrence rates in patients with paroxysmal $\mathrm{AF}$ complicated with severe AIT warrant further studies.

\section{Conclusions}

For patients with paroxysmal AF complicated with AIT, early ablation appears safe and effective when performed under the guidance of a 3-dimensional mapping system, with 30 min observation and use of isoproterenol and pacing to induce atrial tachyarrhythmias related to pulmonary vein and extra-pulmonary vein for ablation.

\section{Conflict of interest: None declared}

\section{References}

1. Hauser TH, Pinto DS, Josephson ME, Zimetbaum P. Early recurrence of arrhythmia in patients taking amiodarone or class $1 \mathrm{C}$ agents for treatment of atrial fibrillation or atrial flutter. Am J Cardiol, 2004; 93: 1173-1176.

2. Wilke T, Groth A, Mueller S et al. Incidence and prevalence of atrial fibrillation: An analysis based on 8.3 million patients. Europace, 2013; 15: 486-493. doi: 10.1093/europace/eus333. 
3. Cohen-Lehman J, Dahl P, Danzi S, Klein I. Effects of amiodarone therapy on thyroid function. Nat Rev Endocrinol, 2010; 6: 34-41, doi:10.1038/nrendo.2009.225.

4. Martino E, Bartalena L, Bogazzi F, Braverman LE. The effects of amiodarone on the thyroid. Endocr Rev, 2001; 22: 240-254.

5. January CT, Wann LS, Alpert JS et al. 2014 AHA/ACC/HRS guideline for the management of patients with atrial fibrillation: Executive summary: A report of the American College of Cardiology/American Heart Association Task Force on practice guidelines and the Heart Rhythm Society. Circulation, 2014; 130: 2071-2104. doi: 10.1161/CIR.0000000000000040.

6. Cardenas GA, Cabral JM, Leslie CA. Amiodarone induced thyrotoxicosis: diagnostic and therapeutic strategies. Cleve Clin J Med, 2003; 70: 624-626, 628-631.

7. Oral H, Scharf C, Chugh A et al. Catheter ablation for paroxysmal atrial fibrillation: segmental pulmonary vein ostial ablation versus left atrial ablation. Circulation, 2003; 108: 2355-2360.

8. Ouyang F, Bansch D, Ernst S et al. Complete isolation of left atrium surrounding the pulmonary veins: New insights from the double-Lasso technique in paroxysmal atrial fibrillation. Circulation, 2004; 110: 2090-2096.

9. Bertaglia E, Tondo C, De Simone A et al. Does catheter ablation cure atrial fibrillation? Single-procedure outcome of drug-refractory atrial fibrillation ablation: A 6-year multicentre experience. Europace, 2010; 12: 181-187. doi: 10.1093/europace/eup349.

10. Arruda M, Mlcochova H, Prasad SK et al. Electrical isolation of the superior vena cava: An adjunctive strategy to pulmonary vein antrum isolation improving the outcome of $\mathrm{AF}$ ablation. J Cardiovasc Electrophysiol, 2007; 18: 1261-1266.

11. Jais P, Hocini M, O'Neill MD et al. How to perform linear lesions. Heart Rhythm, 2007; 4: 803-809.

12. Knecht S, Hocini M, Wright $\mathrm{M}$ et al. Left atrial linear lesions are required for successful treatment of persistent atrial fibrillation. Eur Heart J, 2008; 29: 2359-2366. doi: 10.1093/eurheartj/ehn302.
13. Bartalena L, Brogioni S, Grasso L, Bogazzi F, Burelli A, Martino E. Treatment of amiodarone-induced thyrotoxicosis, a difficult challenge: Results of a prospective study. J Clin Endocrinol Metab, 1996; 81: 2930-2933.

14. Bogazzi F, Bartalena L, Cosci C et al. Treatment of type II amiodarone-induced thyrotoxicosis by either iopanoic acid or glucocorticoids: A prospective, randomized study. J Clin Endocrinol Metab, 2003; 88: 1999-2002.

15. Bogazzi F, Bartalena L, Tomisti L et al. Glucocorticoid response in amiodarone-induced thyrotoxicosis resulting from destructive thyroiditis is predicted by thyroid volume and serum free thyroid hormone concentrations. J Clin Endocrinol Metab, 2007; 92: 556-562.

16. Sakaguchi Y, Cui G, Sen L. Acute effects of thyroid hormone on inward rectifier potassium channel currents in guinea pig ventricular myocytes. Endocrinology, 1996; 137: 4744-4751.

17. Jayachandran JV, Sih HJ, Winkle W, Zipes DP, Hutchins GD, Olgin JE. Atrial fibrillation produced by prolonged rapid atrial pacing is associated with heterogeneous changes in atrial sympathetic innervation. Circulation, 2000; 101: 1185-1191.

18. Bertaglia E, Stabile G, Senatore G et al. Predictive value of early atrial tachyarrhythmias recurrence after circumferential anatomical pulmonary vein ablation. Pacing Clin Electrophysiol, 2005; 28: 366-371.

19. Auer J, Scheibner P, Mische T, Langsteger W, Eber O, Eber B. Subclinical hyperthyroidism as a risk factor for atrial fibrillation. Am Heart J, 2001; 142: 838-842.

20. Ma CS, Liu X, Hu FL et al. Catheter ablation of atrial fibrillation in patients with hyperthyroidism. J Interv Card Electrophysiol, 2007; 18: 137-142.

21. Elayi CS, Di Biase L, Bai R et al. Administration of isoproterenol and adenosine to guide supplemental ablation after pulmonary vein antrum isolation. J Cardiovasc Electrophysiol, 2013; 24: 1199-1206. doi: 10.1111/jce.12252. 